\title{
Magnetization Reversal Time in Ferromagnetic and Ferrimagnetic Materials
}

\author{
T. Kobayashi, and Y. Fujiwara \\ Graduate School of Engineering, Mie Univ., 1577 Kurimamachiya-cho, Tsu 514-8507, Japan
}

Magnetization reversal time in ferrimagnetic material is compared with that in ferromagnetic material. Since ferromagnetic material is in a state of underdamping, the reversal time is relatively long. A formula for reversal time in ferrimagnetic material is deduced under the assumption that the effective Gilbert damping parameter $\alpha_{\text {eff }}$ is inversely proportional to the net angular momentum. In the case of $g_{1}=g_{2}$, where $g_{1}$ and $g_{2}$ are the g-factors of the first and second sublattices, respectively, the reversal time in ferrimagnetic material is much shorter than that in ferromagnetic material because $\alpha_{\text {eff }}$ in ferrimagnetic material can be adjusted so that $\alpha_{\text {eff }}=1$. Furthermore, in the case of $g_{1} \neq g_{2}$, the reversal time becomes much shorter than that when $g_{1}=g_{2}$ because a large net magnetization and small net angular momentum can be realized.

Key words: magnetization reversal time, ferrimagnetic material, damping parameter, g-factor, angular momentum

\section{Introduction}

High-density magnetic recording requires a short time for writing due to the tiny mark size. For example, the time for writing is only $10^{-1} \mathrm{~ns}$ for a few Tbpsi at a rotation speed $10,000 \mathrm{rpm}$ in a 3.5 -inch hard disk drive. A new problem therefore arises as to whether the reversal time of magnetization is sufficiently short. In this paper, we discuss the possibility of high-speed reversal of magnetization in ferrimagnetic material in comparison with that in ferromagnetic material.

\section{Magnetization reversal time in ferromagnetic}

\section{material}

It is well known that the magnetization reversal time $\tau$ in ferromagnetic material can be calculated by

$$
\tau=\frac{1+\alpha^{2}}{\alpha} \frac{1}{|\gamma|} \frac{1}{H_{\mathrm{ext}}},
$$

where $\alpha,|\gamma|$, and $H_{\text {ext }}$ are the Gilbert damping parameter, the gyromagnetic ratio, and the external magnetic field, respectively. Under an anisotropic field $H_{\mathrm{k}}$, the equation should be modified as follows:

$$
\begin{aligned}
& \tau^{\prime}=\frac{1+\alpha^{2}}{\alpha} \frac{1}{|\gamma|}\left(\frac{1}{2\left(H_{\mathrm{ext}}-H_{\mathrm{k}}\right)}+\frac{1}{2\left(H_{\mathrm{ext}}+H_{\mathrm{k}}\right)}\right) \\
& =\frac{1+\alpha^{2}}{\alpha} \frac{1}{|\gamma|} \frac{H_{\mathrm{ext}}}{\left(H_{\mathrm{ext}}^{2}-H_{\mathrm{k}}^{2}\right)}>\tau,
\end{aligned}
$$

\section{in which $\tau^{\prime}$ is longer than $\tau$.}

The results of calculating $\tau^{\prime}$ under the assumption that the Gilbert damping parameter $\alpha=0.04{ }^{1)}$ and the gyromagnetic ratio $|\gamma|=1.76 \times 10^{7} 1 /(\mathrm{Oe} \cdot \mathrm{s})$ are shown in Fig. 1. It is necessary for the fast reversal of magnetization that $H_{\mathrm{ext}}$ be sufficiently larger than $H_{\mathrm{k}}$.

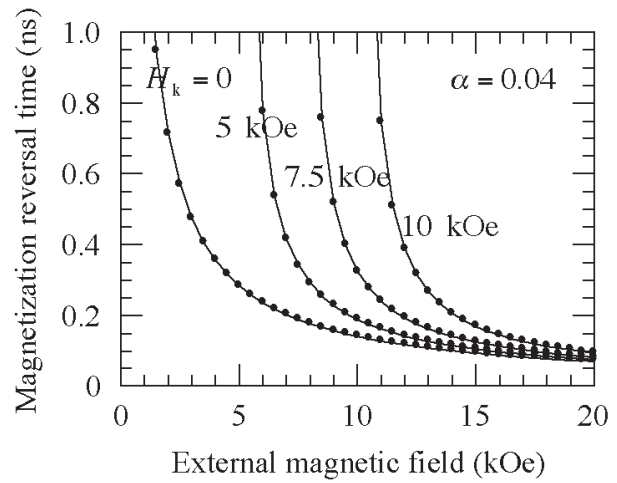

Fig. 1 Magnetization reversal time as a function of the external magnetic field in ferromagnetic material.

Considering the limitation on the head field $H_{\text {ext }}$ and the thermal stability determined by the magnetic anisotropy $H_{\mathrm{k}}$, the reversal time may be comparable with the writing time of $10^{-1} \mathrm{~ns}$ for high-density recording. Since $\alpha=0.04$, the motion of the magnetization is considerable to be underdamping. If we can control the value of $\alpha$ and increase the critical damping up to $\alpha=1, \tau^{\prime}$ can be decreased. However, such a mean is unknown. One possibility to avoid the above problem may be the employment of ferrimagnetic materials. The details are explained below.

\section{Magnetization reversal time in ferrimagnetic}

\section{material}

\subsection{Effective Gilbert damping parameter}

A ferrimagnetic material has two sublattice magnetizations $M_{\mathrm{si}}$ and corresponding angular momentums $A_{\mathrm{s} i}=M_{\mathrm{si}}|| \gamma_{i} \mid$. The net magnetization is $M_{\mathrm{s}}=\left|M_{\mathrm{s} 1}-M_{\mathrm{s} 2}\right|$, and the net angular momentum is $A_{\mathrm{s}}=\left|A_{\mathrm{s} 1}-A_{\mathrm{s} 2}\right|$. Both the gyromagnetic ratio $\left|\gamma_{i}\right|$ and the $\mathrm{g}$-factor $g_{i}$ are the ratio of the magnetization to the angular momentum; that is, $\left|\gamma_{i}\right|=M_{\mathrm{s} i} / A_{\mathrm{s} i}=g_{i}\left(\mu_{\mathrm{B}} / \hbar\right)$, where $\mu_{\mathrm{B}}$ is the Bohr magneton. Therefore, when the 


$$
\sum_{A_{\mathrm{s} 1}}^{T=T_{\mathrm{M}}}+\overbrace{A_{\mathrm{s} 2}}^{M_{\mathrm{s} 2}}={ }_{A_{\mathrm{s}}}=0
$$

(a)

$$
M_{\mathrm{s} 1}^{T=T_{\mathrm{A}}}+\overbrace{A_{\mathrm{s} 2}}^{M_{\mathrm{s} 2}}=M_{\mathrm{s}}=0
$$

(b)

Fig. 2 Configurations of magnetizations and angular momentums at (a) magnetization compensation temperature $T_{\mathrm{M}}$ and (b) angular momentum compensation temperature $T_{\mathrm{A}}$ for the case of $g_{1} \neq g_{2}$.

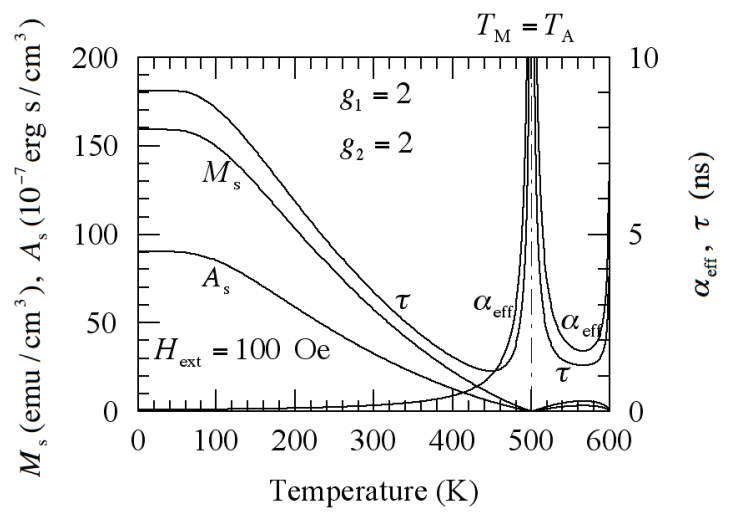

Fig. 3 Temperature dependence of net magnetization $M_{\mathrm{s}}$, net angular momentum $A_{\mathrm{s}}$, effective Gilbert damping parameter $\alpha_{\text {eff }}$, and reversal time $\tau$ of magnetization in ferrimagnetic material for the case of $g_{1}=g_{2}=2$. The material of $g_{1}=g_{2}$ is hypothetical.

g-factor $g_{1}$ of the first sublattice is different from the g-factor $g_{2}$ of the second sublattice, the magnetization compensation temperature $T_{\mathrm{M}}$ does not agree with the angular momentum compensation temperature $T_{\mathrm{A}}$. Although $M_{\mathrm{s}}=0$ at $T_{\mathrm{M}}, A_{\mathrm{s}}$ has a non-zero value in such a case (Fig. $2(\mathrm{a})$ ). Similarly, although $A_{\mathrm{s}}=0$ at $T_{\mathrm{A}}, M_{\mathrm{s}}$ has a non-zero value (Fig. $2(\mathrm{~b})$ ).

We have proposed that the effective Gilbert damping parameter $\alpha_{\text {eff }}$ in ferrimagnetic material is inversely proportional to $A_{\mathrm{s}}$; that is, $\alpha_{\text {eff }}=A_{0} / A_{\mathrm{s}}{ }^{2}$, where $A_{0}$ is defined as $\lambda_{1} /\left|\gamma_{1}\right|^{2}+\lambda_{2} /\left|\gamma_{2}\right|^{2}$ using the Landau-Lifshitz damping parameter $\lambda_{i}$, and $A_{0}$ is assumed to be constant. This assumption was confirmed by an experiment conducted by C. D. Stanciu et al. ${ }^{3)}$. It is also well known that the effective gyromagnetic ratio $\gamma_{\text {eff }}$ is expressed as $\gamma_{\text {eff }}=M_{\mathrm{s}} / A_{\mathrm{s}}$. Our calculations in this paper are made under the above assumptions.

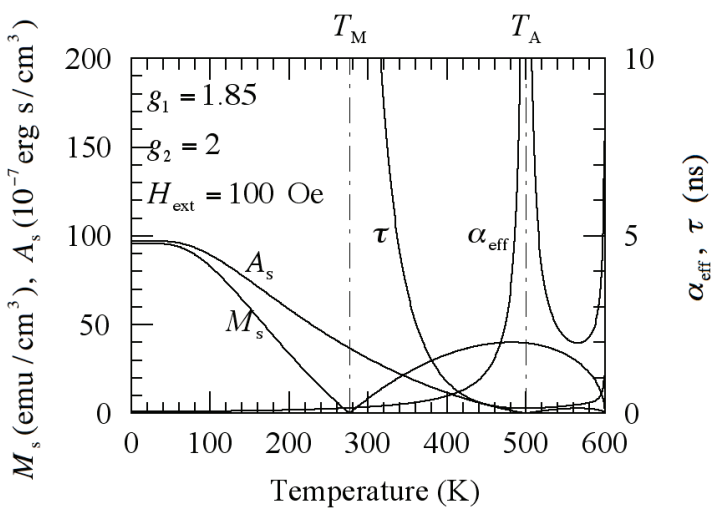

Fig. 4 Temperature dependence of $M_{\mathrm{s}}, A_{\mathrm{s}}, \alpha_{\text {eff }}$, and $\tau$ of magnetization in ferrimagnetic material for the case of $g_{1}=1.85$ and $g_{2}=2$. The g-factors $g_{1}=1.85$ and $g_{2}=2$ correspond the alloy of Gd-Tb-Fe-Co.

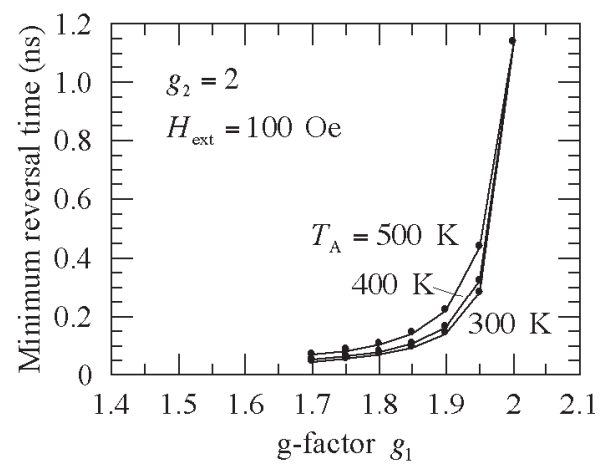

Fig. 5 Minimum reversal time as a function of the g-factor $g_{1}$.

\subsection{Case of g-factor $g_{1}=g_{2}$}

Substituting $\alpha_{\text {eff }}$ for $\alpha$ and $\gamma_{\text {eff }}$ for $|\gamma|$ in Eq. (1), we obtain the magnetization reversal time $\tau$ for ferrimagnetic materials as

$$
\tau=\frac{1+\alpha_{\mathrm{eff}}^{2}}{\alpha_{\mathrm{eff}}} \frac{1}{\gamma_{\mathrm{eff}}} \frac{1}{H_{\mathrm{ext}}}
$$

Since $M_{\mathrm{s}}$ is proportional to $A_{\mathrm{s}}$ in the case of $g_{1}=g_{2}, \quad \gamma_{\text {eff }}$ becomes constant and $T_{\mathrm{M}}$ agrees with $T_{\mathrm{A}}$. Hence, $\tau$ reaches a minimum when $\alpha_{\text {eff }}=1$.

The damping parameter $\alpha$ has often been assumed to be a constant. This assumption, however, gives rise to the inconsistency that the magnetization $M_{\mathrm{s}}$ can be reversed even when $M_{\mathrm{s}}=0$ at $T_{\mathrm{M}}$ from Eq. (3). In our assumption, $\alpha_{\text {eff }}$ is inversely proportional to $M_{\mathrm{s}}$ when $g_{1}=g_{2}$. Therefore, $\tau$ is expressed as follows:

$$
\tau \propto \frac{M_{\mathrm{s}}^{2}+\text { const. }}{M_{\mathrm{s}}}
$$

From Eq. (4), we obtain a consistent interpretation that $\tau \rightarrow \infty$ when $M_{\mathrm{s}}=0$.

A simulation was carried out by the ordinary mean field method using ferrimagnetic material of a rare earth-3d transition metal such as Gd-Fe or Gd-Co. 
Strictly speaking, although $g_{1}$ and $g_{2}$ of $\mathrm{Gd}-\mathrm{Fe}$ or Gd-Co are almost identical, they are not completely in agreement because the experimental g-factor of $\mathrm{Gd}-\mathrm{Fe}$ or Gd-Co shows temperature dependence. Therefore the material of $g_{1}=g_{2}$ is hypothetical. The calculation parameters were $\left.A_{0}=5.69 \times 10^{-7} \mathrm{erg} \cdot \mathrm{s} / \mathrm{cm}^{3}{ }^{2}\right), H_{\text {ext }}=$ $100 \mathrm{Oe}$, and a Curie temperature of $600 \mathrm{~K}$. The reversal time was found to considerably vary with the temperature, as shown in Fig. 3. It can be seen that $\tau$ is divergent at $T_{\mathrm{M}}$. The damping parameter $\alpha_{\text {eff }}$ becomes $\alpha_{\text {eff }}=1$ near the point at which $T_{\mathrm{M}}=T_{\mathrm{A}}$. The minimum reversal time $\tau_{\min }$ is $1.14 \mathrm{~ns}$, which can be compared with the value of $14.2 \mathrm{~ns}$ in ferromagnetic material. The reversal time in ferrimagnetic material is expected to be one order faster than that in ferromagnetic material.

\subsection{Case of $g$-factor $g_{1} \neq g_{2}$}

In the case of $g_{1} \neq g_{2}$, substituting $\alpha_{\text {eff }}=A_{0} / A_{\mathrm{s}}$ for $\alpha$ and $\gamma_{\text {eff }}=M_{\mathrm{s}} / A_{\mathrm{s}}$ for $|\gamma|$ in Eq. (1), $\tau$ is expressed as

$$
\tau=\frac{1+\alpha_{\mathrm{eff}}{ }^{2}}{\alpha_{\mathrm{eff}}} \frac{1}{\gamma_{\mathrm{eff}}} \frac{1}{H_{\mathrm{ext}}}=\frac{A_{\mathrm{s}}{ }^{2}+A_{0}{ }^{2}}{A_{0}} \frac{1}{M_{\mathrm{s}} H_{\mathrm{ext}}} .
$$

Since the magnetization $M_{\mathrm{s}}$ is proportional to $A_{\mathrm{s}}$ in ferromagnetic material, $M_{\mathrm{s}}$ and $A_{\mathrm{s}}$ cannot be controlled independently. In ferrimagnetic material, on the other hand, when $g_{1} \neq g_{2}$, a state in which $M_{\mathrm{s}}$ is large and $A_{\mathrm{s}}$ is small can be realized. The term $M_{\mathrm{s}} H_{\mathrm{ext}}$ is the energy of magnetization reversal in Eq. (5), and $A_{\mathrm{s}}$ is the cause of precession. It is interpreted from Eq. (5) that the precession caused by $A_{\mathrm{s}}$ makes the reversal time long. A large $M_{\mathrm{s}} H_{\mathrm{ext}}$ and small $A_{\mathrm{s}}$ consequently bring about a short reversal time of magnetization. Furthermore, from Eq. (5) it is also seen that a small $A_{0}$ makes the reversal time short under the condition of $A_{\mathrm{s}}=0$. The term $A_{0}$ may thus be regarded as a type of friction.

In addition, we can also obtain the same interpretation that $\tau \rightarrow \infty$ when $M_{\mathrm{s}}=0$ from Eq. (5).

When $g_{1}=1.85$ and $g_{2}=2, \tau_{\text {min }}$ is only $0.14 \mathrm{~ns}$ around $T_{\mathrm{A}}$ due to the large $M_{\mathrm{s}}$ and small $A_{\mathrm{s}}$, as shown in Fig. 4, compared with the value of $1.14 \mathrm{~ns}$ for the case of $g_{1}=g_{2}$. The g-factors $g_{1}=1.85$ and $g_{2}=2$ correspond to the alloy of $\mathrm{Gd}-\mathrm{Tb}-\mathrm{Fe}-\mathrm{Co}$ since $g_{1}$ of $\mathrm{Gd}$ is 2 and $g_{1}$ of $\mathrm{Tb} 3 / 2$.

Figure 5 shows the minimum reversal time $\tau_{\text {min }}$ as a function of the g-factor $g_{1}$. When $g_{1}$ decreases from $g_{1}=2, \tau_{\min }$ decreases rapidly. To decrease $g_{1}$, the use of low g-factor element, such as $\mathrm{Tb}, \mathrm{Dy}$, Ho, may be effective.

\section{Discussion}

Even if $g_{1}=g_{2}$, the reversal time in ferrimagnetic material is one order faster than that in ferromagnetic material. From the relationships of $\alpha=A_{0} / A_{\mathrm{s}}$ and
$|\gamma|=M_{\mathrm{s}} / A_{\mathrm{s}}$, we obtain $A_{0}=\alpha M_{\mathrm{s}} /|\gamma|$. In this paper, we assumed $\alpha=0.04$ and $|\gamma|=1.76 \times 10^{7} 1 /(\mathrm{Oe} \cdot \mathrm{s}) \quad$ for ferromagnetic material. We can estimate the value of $A_{0}$ in ferromagnetic material by the further assumption of $M_{\mathrm{s}}=500 \mathrm{emu} / \mathrm{cm}^{3}$; that is, $A_{0}=11.4 \times 10^{-7} \mathrm{erg} \cdot \mathrm{s} / \mathrm{cm}^{3}$, which is only double the value of $A_{0}=5.69 \times 10^{-7} \mathrm{erg} \cdot \mathrm{s} / \mathrm{cm}^{3}$ in ferrimagnetic material. On the other hand, $\left(1+\alpha^{2}\right) / \alpha$ is 25 for $\alpha=0.04$ in ferromagnetic material. In ferrimagnetic material, $\alpha_{\text {eff }}$ can be adjusted so that $\alpha_{\text {eff }}=1$, and $\left(1+\alpha_{\text {eff }}{ }^{2}\right) / \alpha_{\text {eff }}$ is only 2 when $\alpha_{\text {eff }}=1$. Hence, the main reason for the slow reversal time of ferromagnetic material is the state of underdamping of $\alpha=0.04$.

Furthermore, if $g_{1} \neq g_{2}$, the reversal time becomes much faster than that in the case of $g_{1}=g_{2}$ because a large net magnetization and small net angular momentum can be realized. Since $M_{\mathrm{s}}$ is proportional to $A_{\mathrm{s}}$ in the case of both ferromagnetic and ferrimagnetic materials when $g_{1}=g_{2}$, some precession is essential to reserve the energy $M_{\mathrm{s}} H_{\text {ext }}$ of magnetization reversal. On the other hand, in the case of ferrimagnetic material when $g_{1} \neq g_{2}$, precession is completely unnecessary. In other words, the precession caused by the net angular momentum makes the reversal time long. However, the magnetization can be reversed without the net angular momentum when $g_{1} \neq g_{2}$. Thus, high-speed magnetization reversal is possible.

Although the exchange mode is not taken into consideration, the role of the magnetization and the angular momentum in the magnetization reversal time for ferrimagnetic material becomes clear in this calculation.

\section{Conclusion}

The magnetization reversal time in ferromagnetic material was calculated. Considering the limitation on the head field and thermal stability determined by magnetic anisotropy, the reversal time may be comparable with the writing time for high-density recording.

One possible means of avoiding this problem may be the employment of ferrimagnetic materials. When the g-factor of the first sublattice is different from that of the second sublattice, a large net magnetization and small net angular momentum can be realized. This state is effective for high-speed reversal of magnetization. A one or two order faster reversal time can be estimated for ferrimagnetic material in comparison with ferromagnetic material.

Acknowledgements The authors acknowledge the support of the Storage Research Consortium (SRC), Japan.

\section{References}

1) N. Inaba, Y. Uesaka, A. Nakamura, M. Futamoto, Y. Sugita, and S. Narishige: IEEE Trans. Magn., 33, 2989 (1997). 
2) T. Kobayashi, H. Hayashi, Y. Fujiwara, and S. Shiomi: IEEE Trans. Magn., 41, 2848 (2005).

3) C. D. Stanciu, A. V. Kimel, F. Hansteen, A. Tsukamoto, A. Itoh, A. Kirilyuk, and Th. Rasing: Phys. Rev. B 73, 220402(R) (2006).

Received May 14, 2009; Accepted July 7, 2009 